\title{
Insight into catalytically relevant correlated motions in human purine nucleoside phosphorylase
}

\author{
Sara Núñez \\ Department of Biochemistry, Albert Einstein College of Medicine and \\ Department of Biophysics, Albert Einstein College of Medicine \\ Corin Wing \\ Department of Biochemistry, Albert Einstein College of Medicine \\ Dimitri Antoniou \\ Department of Biophysics, Albert Einstein College of Medicine \\ Vern L. Schramm \\ Department of Biochemistry, Albert Einstein College of Medicine \\ Steven D. Schwartz* \\ Department of Biochemistry, Albert Einstein College of Medicine and \\ Department of Biophysics, Albert Einstein College of Medicine
}

${ }^{*}$ Corresponding author. E-mail: sschwartz@aecom . yu . edu 


\section{SUPPLEMENTARY MATERIAL}

Below, we denote the results of the Essential Dynamics analysis performed in this paper. Figures 1a-3a show the first 9 eigenvectors of the simulations for the apo hPNP model, namely APO, APO2 and APO3. Figures 4a-6a show the first 9 eigenvectors of the simulations for the Michaelis Complex of hPNP model, namely ES, ES2 and ES3. Figures 7a-9a show the first 9 eigenvectors of the simulations for the EI-APO model, that is, the simulation depicting the relaxation of tight complexed hPNP with a TS analog, towards its relaxed apo form, e.i. EI-APO, EI-APO2 and EIAPO3.

Figures 10a-12a show the first 9 eigenvectors of the 1 ns simulations for the APO, ES and EIAPO respectively. These extended $1 \mathrm{~ns}$ simulations were performed for each model in order to certify that the shorter simulations are indeed adequate to capture the types of concerted motions under investigation, i.e. loop closure in hPNP.

Figure 13a shows the first 9 eigenvectors of the solvated simulation for the APO model. Due to the significant increase of CPU cost per dynamics step in explicit solvent, a single solvated simulation under cubic periodic boundary conditions for 200 ps was performed in order to verify that existing motions in the in vacuo systems correlate with the motions found in the solvated complexes. Though the majority of the simulations are performed in vacuum, which inherently enhances flexibility of hydrophobic groups since there is no solvent damping their motions, we expect the analysis of the sampling to provide information that can be carried over to solvated simulations. 


\section{List of Figures}

FIGURE 1a From left to right, and top to bottom, the eigenvectors 1-9 of the APO simulation are shown.

FIGURE 2a From left to right, and top to bottom, the eigenvectors 1-9 of the APO2 simulation are shown.

FIGURE 3a From left to right, and top to bottom, the eigenvectors 1-9 of the APO3 simulation are shown.

FIGURE 4a From left to right, and top to bottom, the eigenvectors 1-9 of the ES simulation are shown.

FIGURE 5a From left to right, and top to bottom, the eigenvectors 1-9 of the ES2 simulation are shown.

FIGURE 6a From left to right, and top to bottom, the eigenvectors 1-9 of the ES3 simulation are shown.

FIGURE 7a From left to right, and top to bottom, the eigenvectors $1-9$ of the EI-APO simulation are shown.

FIGURE 8a From left to right, and top to bottom, the eigenvectors 1-9 of the EI-APO2 simulation are shown.

FIGURE 9a From left to right, and top to bottom, the eigenvectors 1-9 of the EI-APO3 simulation are shown.

FIGURE 10a From left to right, and top to bottom, the eigenvectors 1-9 of the 1 ns APO simulation are shown.

FIGURE 11a From left to right, and top to bottom, the eigenvectors 1-9 of the $1 \mathrm{~ns}$ ES simulation are shown.

FIGURE 12a From left to right, and top to bottom, the eigenvectors 1-9 of the 1 ns EI-APO simulation are shown. 
FIGURE 13a From left to right, and top to bottom, the eigenvectors 1-9 of the solvated APO simulation are shown. 

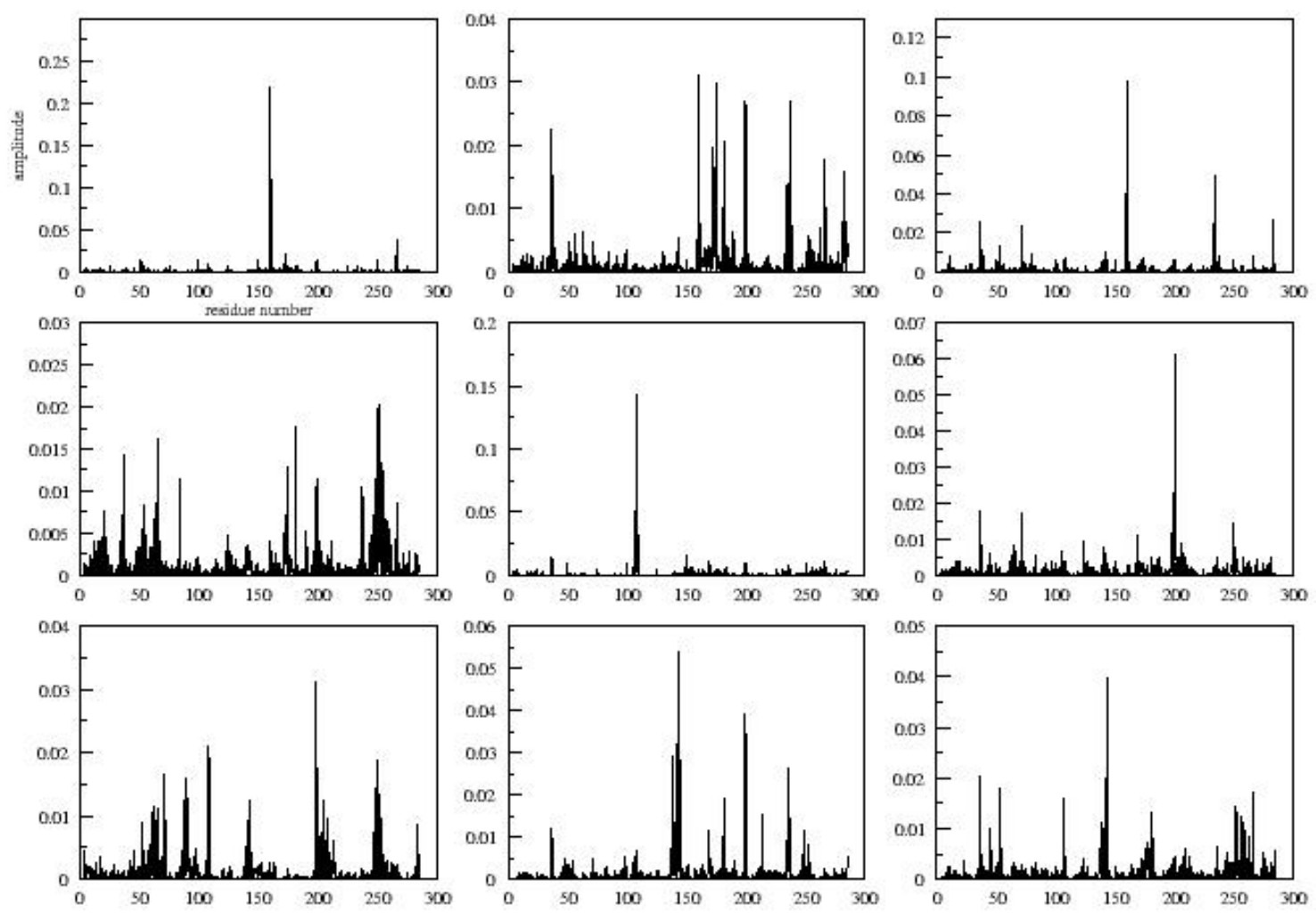

Figure 1a 

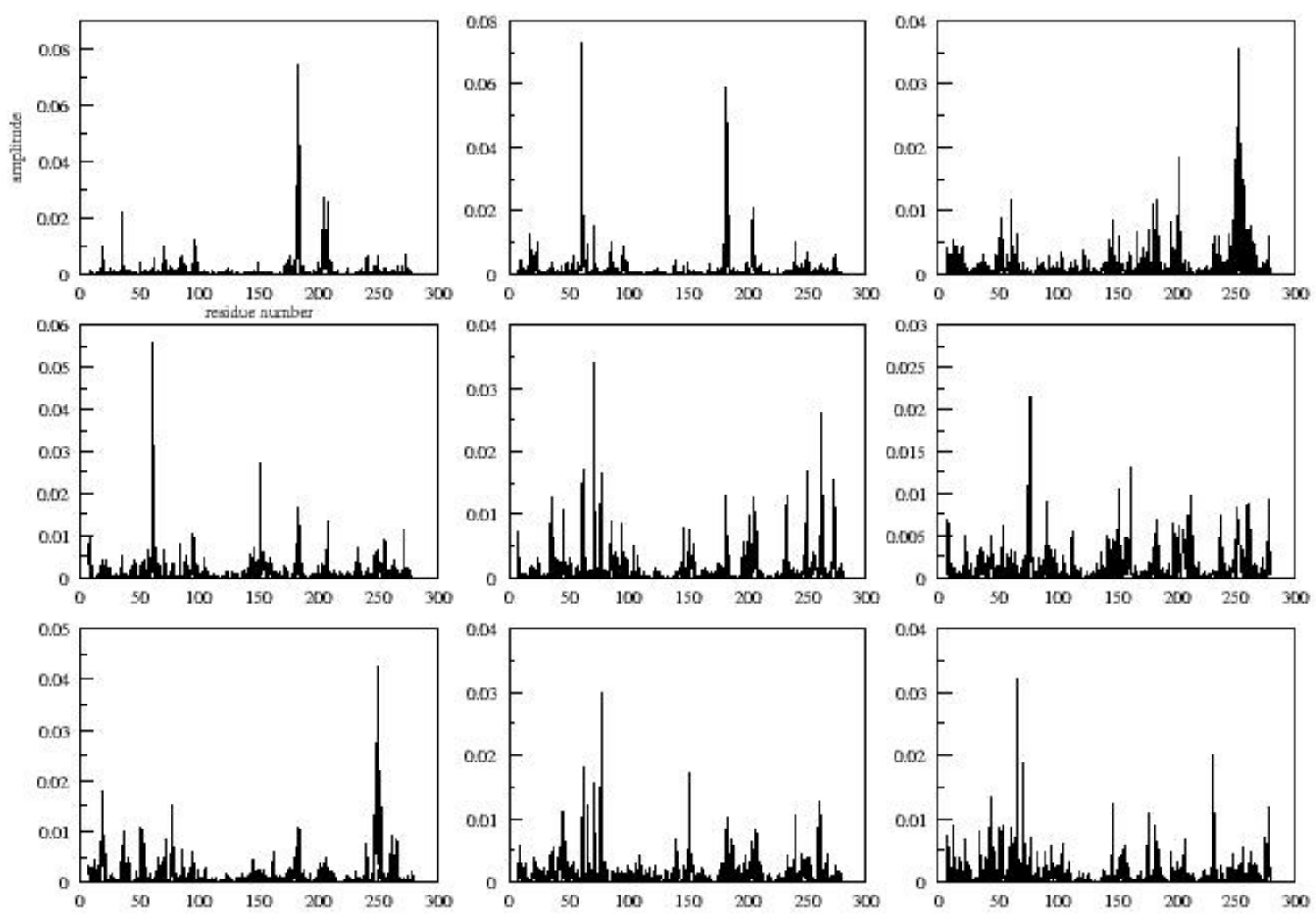

Figure 2a 

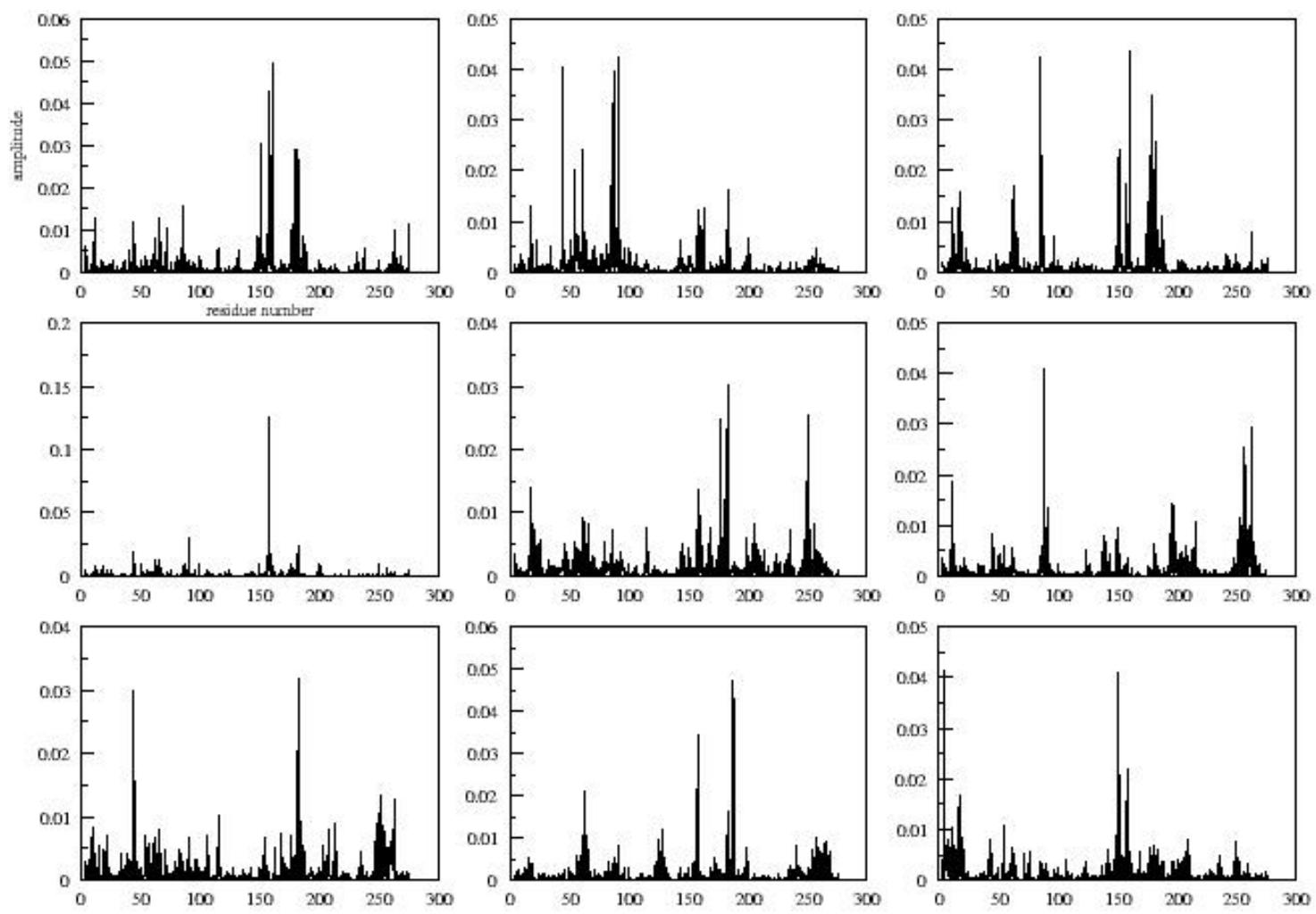

Figure $3 a$ 

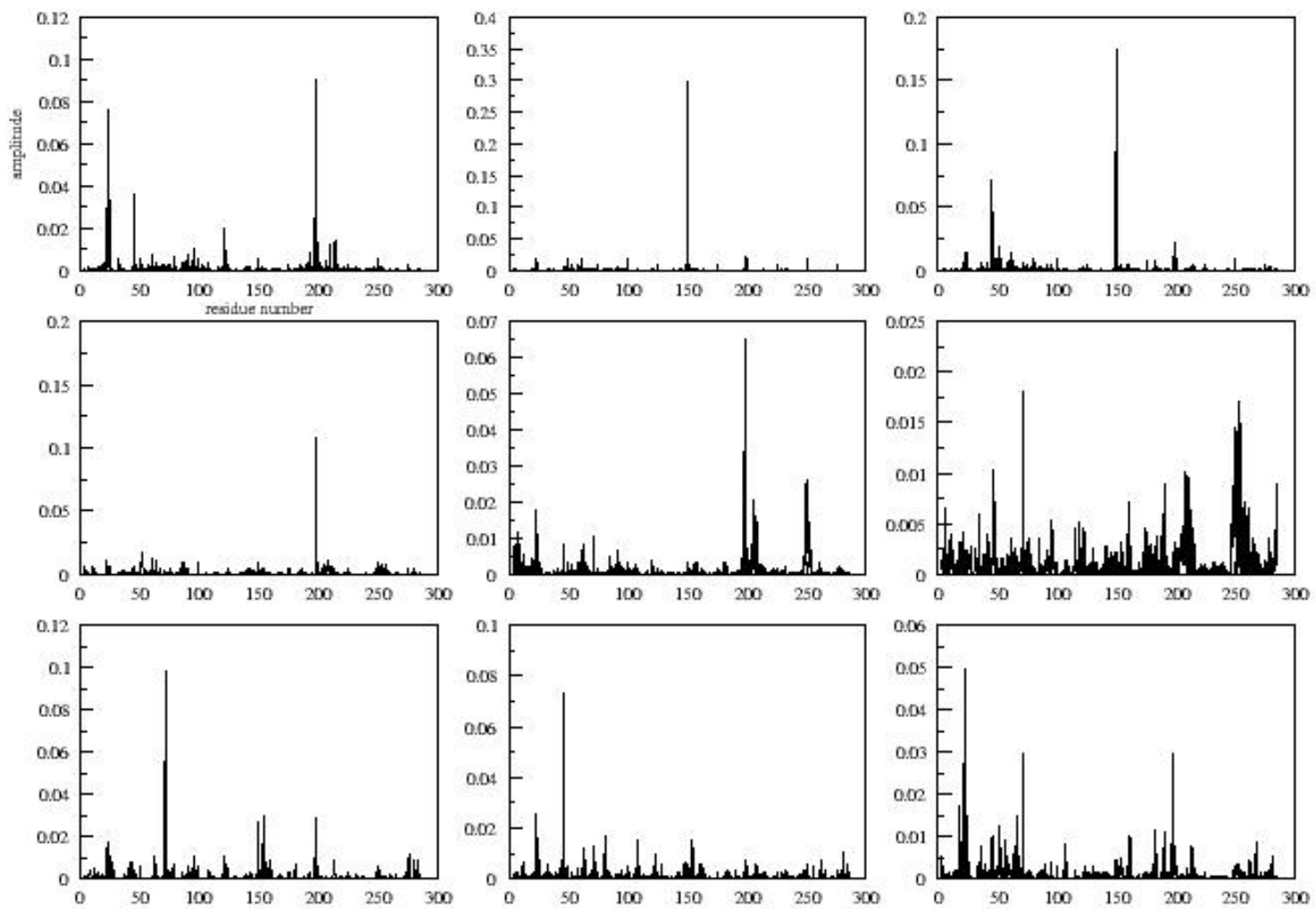

Figure 4a 

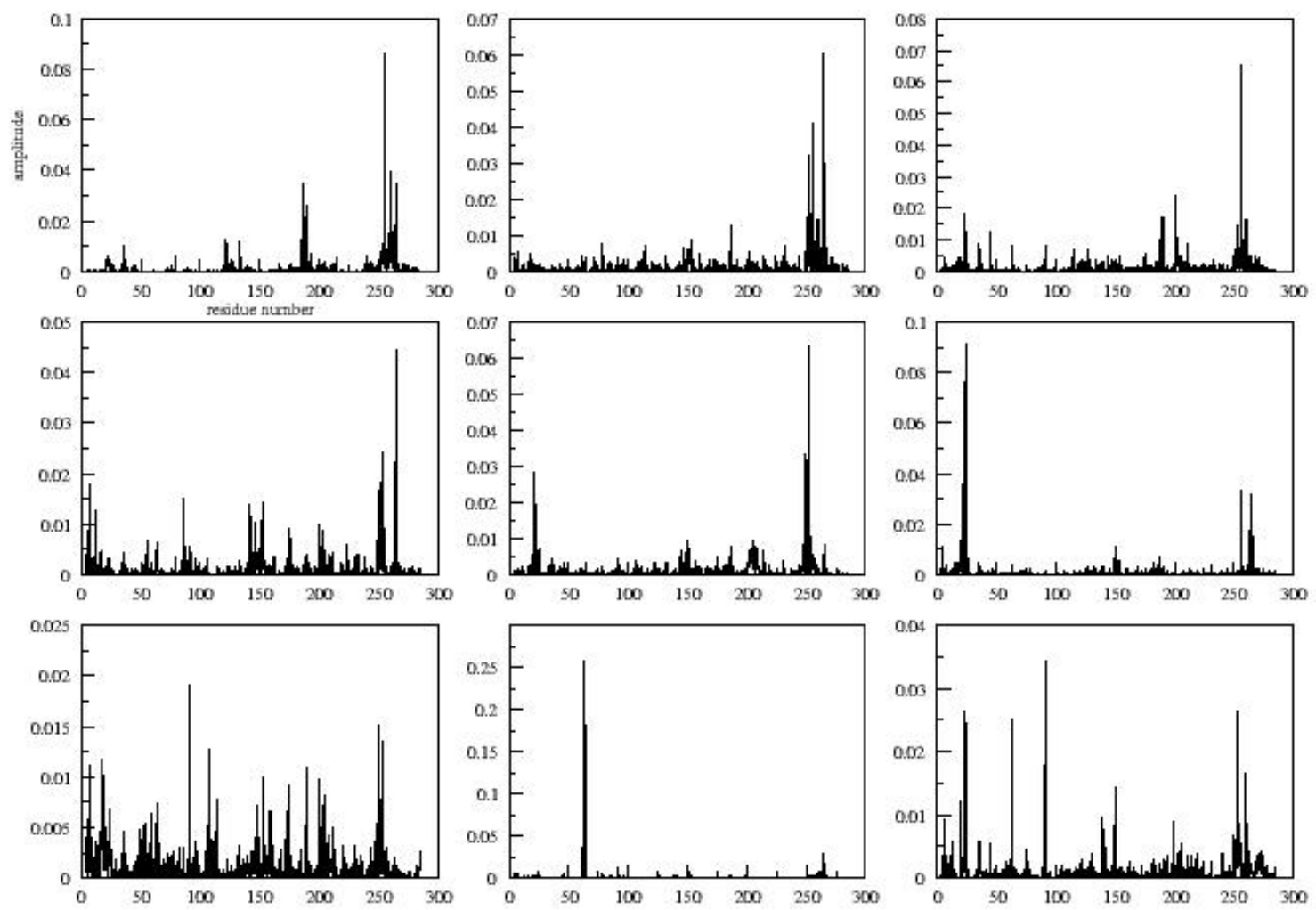

Figure 5a 

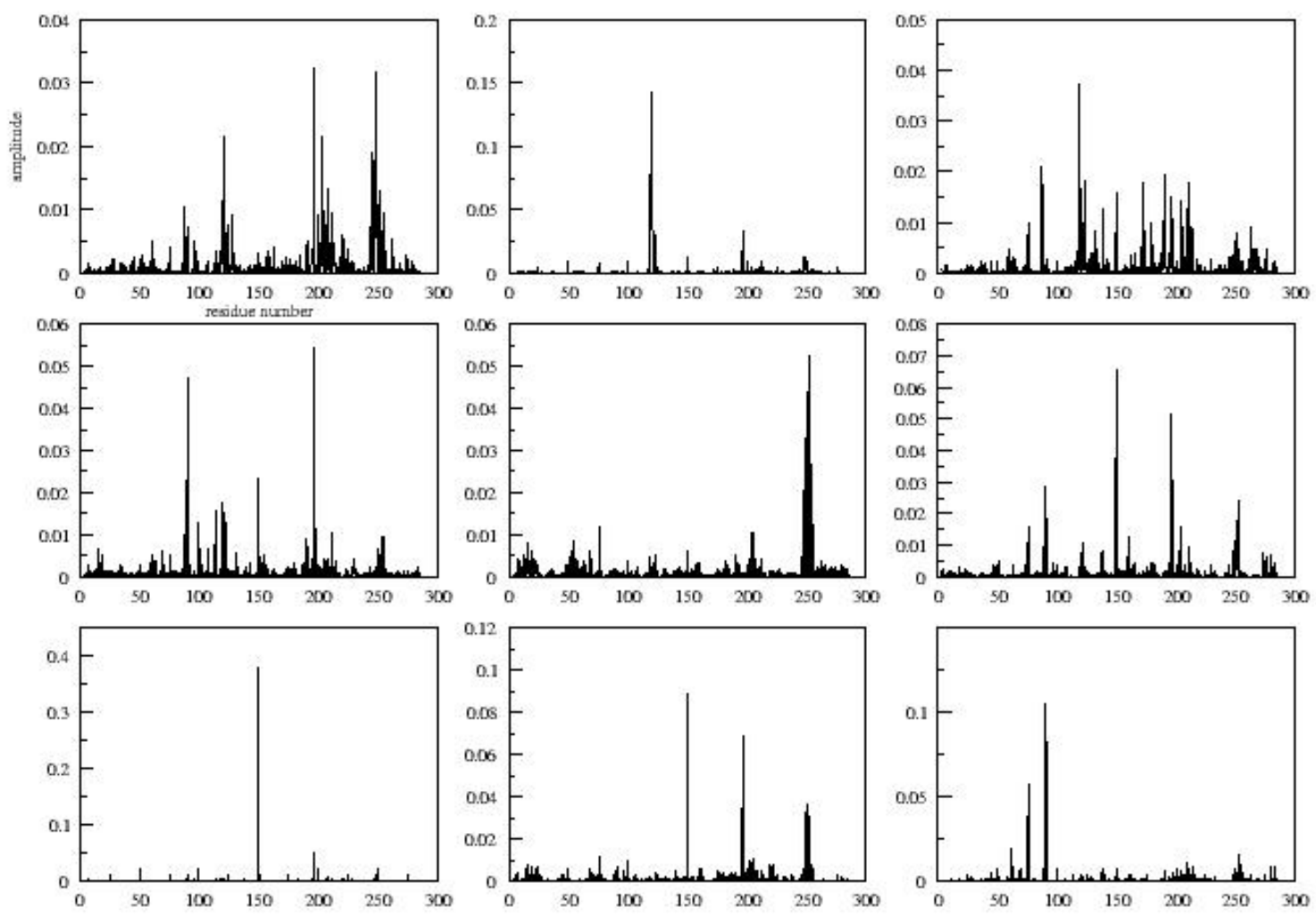

Figure 6a 

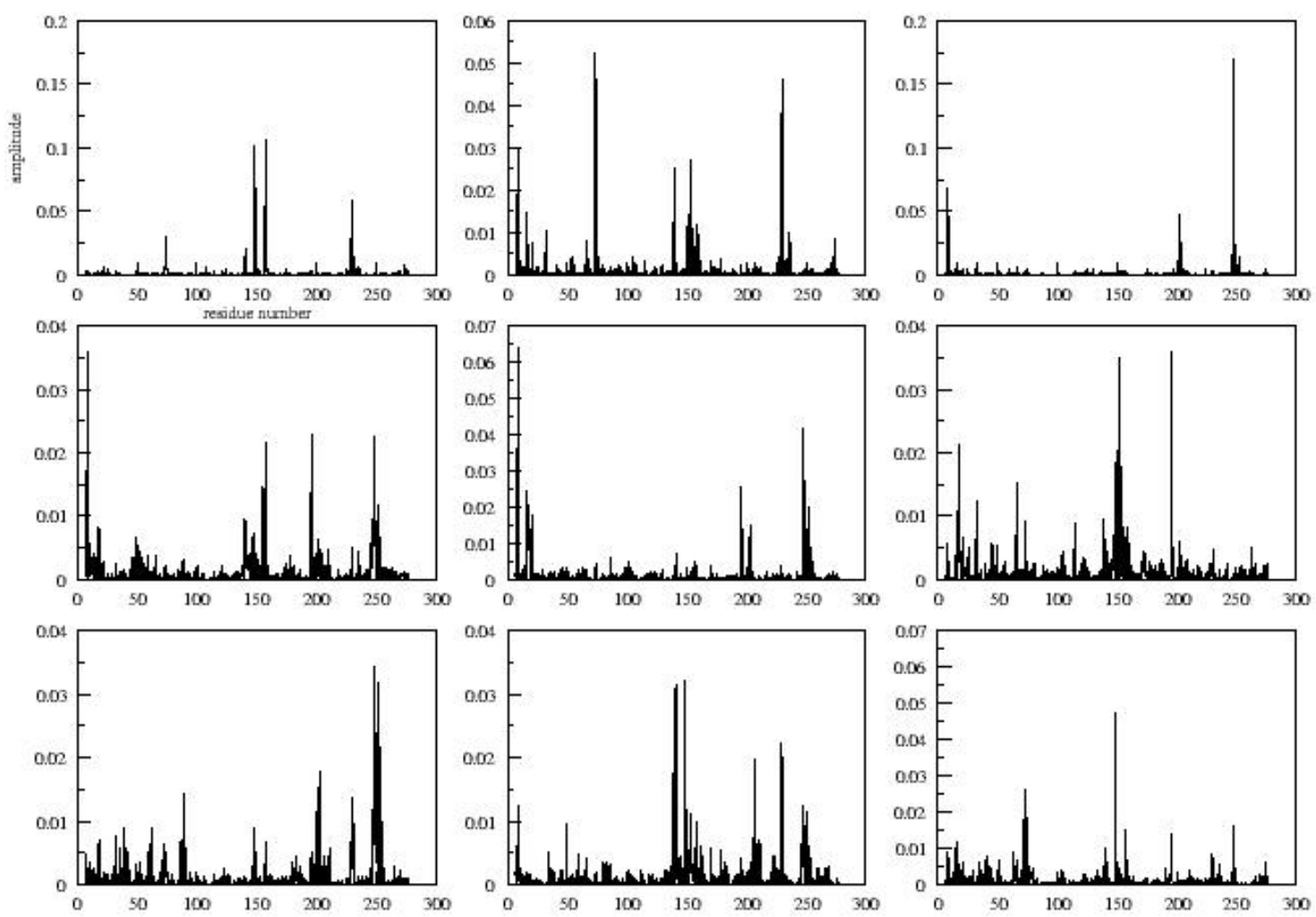

Figure $7 \mathrm{a}$ 

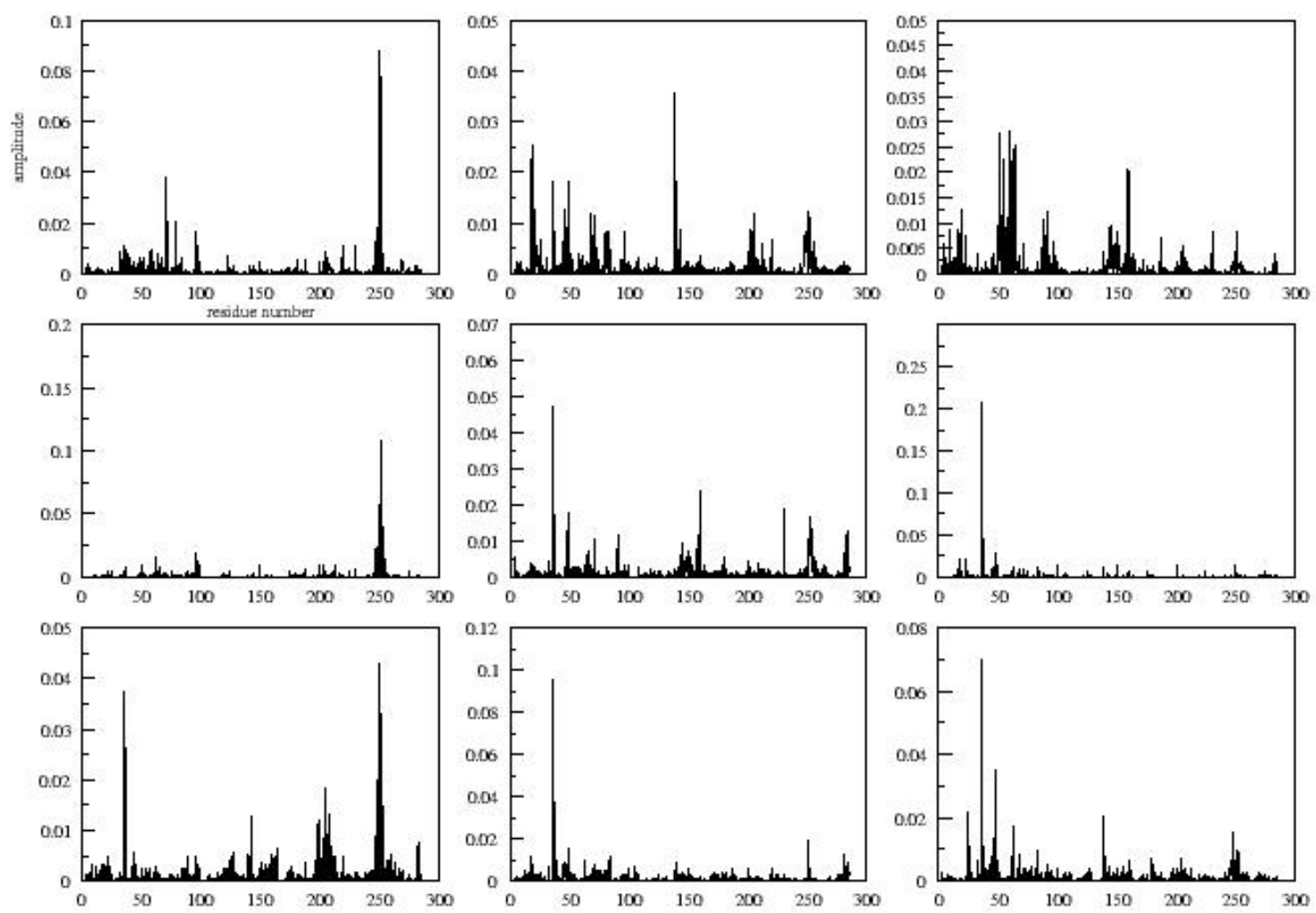

Figure $8 \mathrm{a}$ 

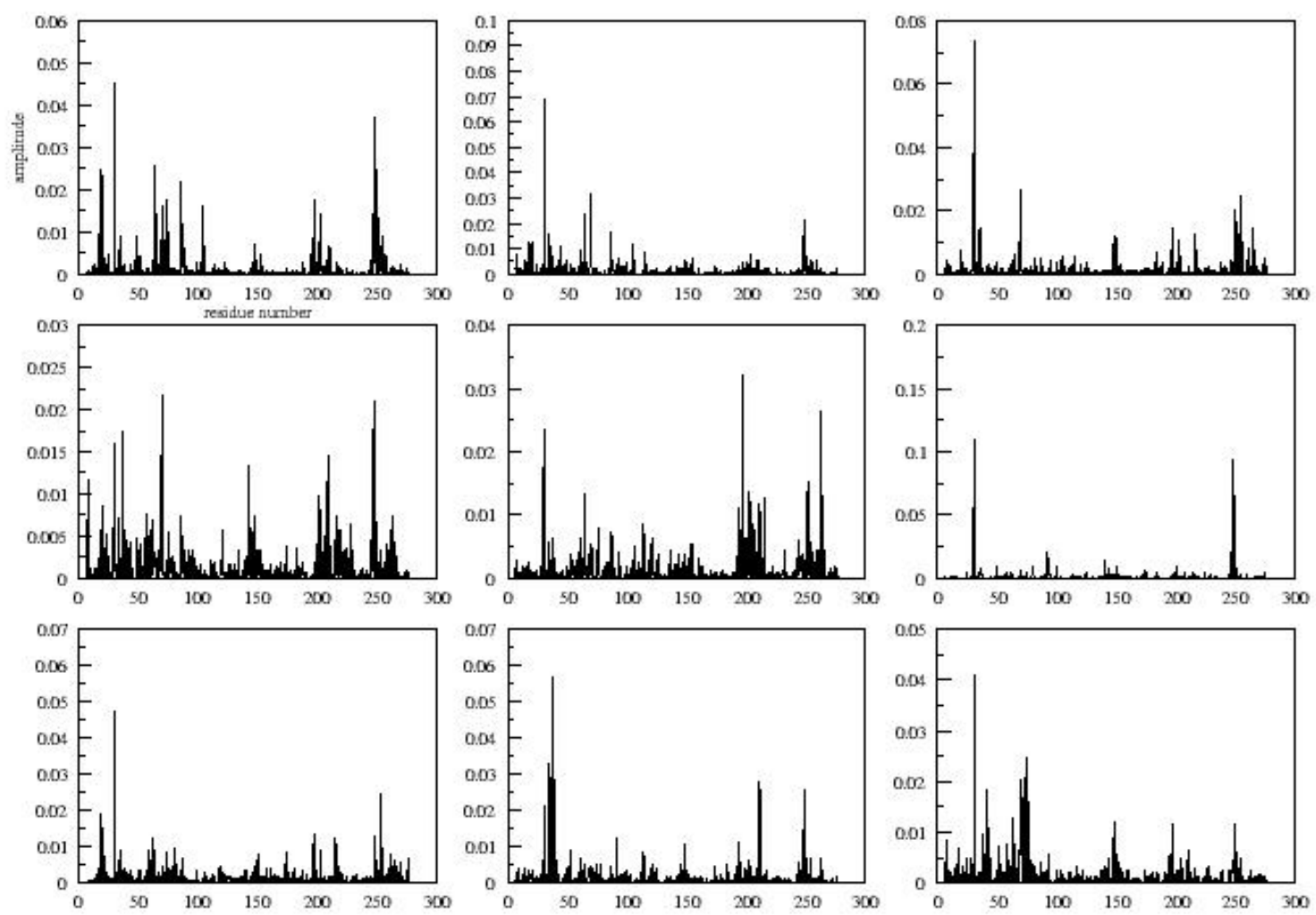

Figure 9a 

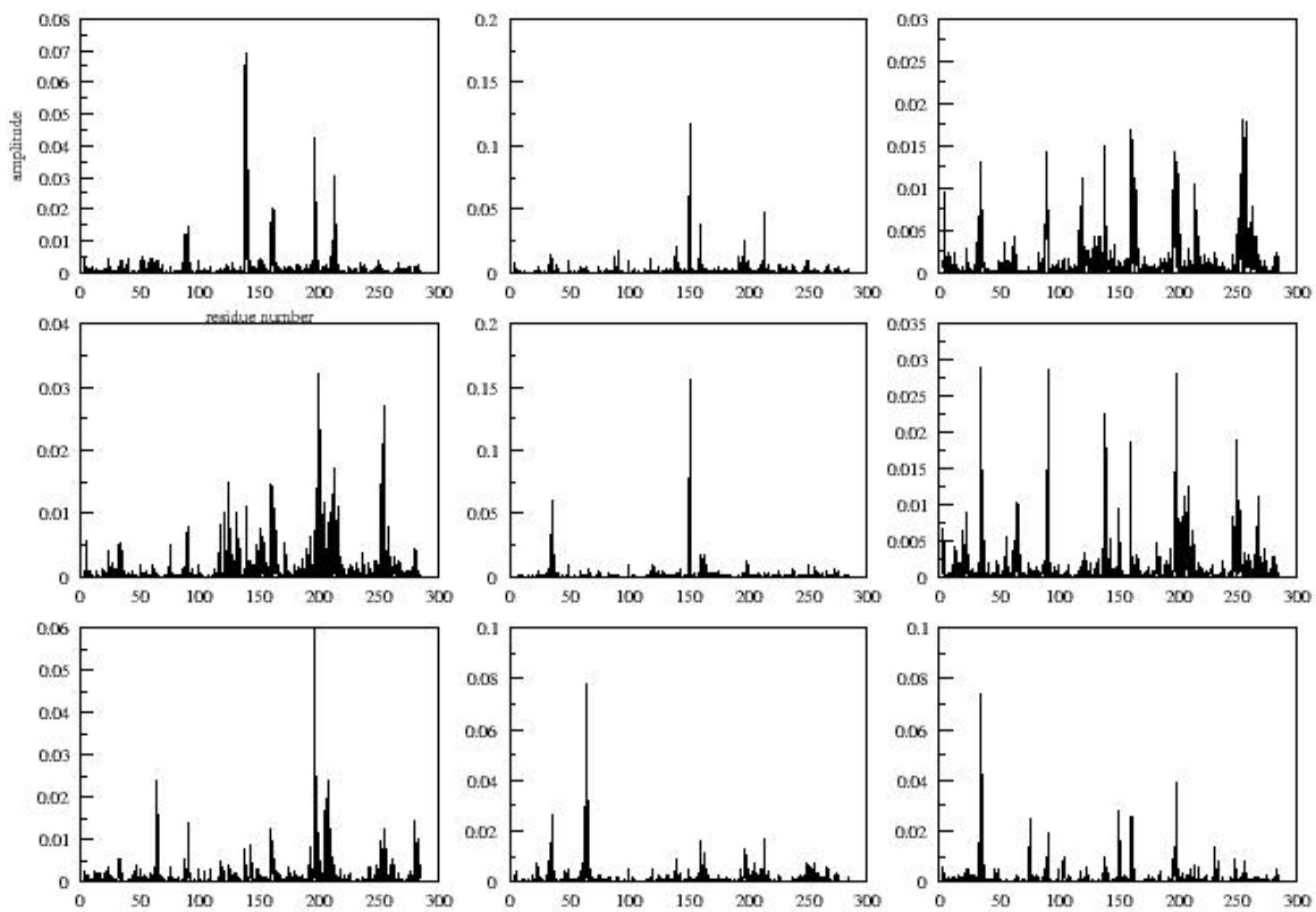

Figure 10a 

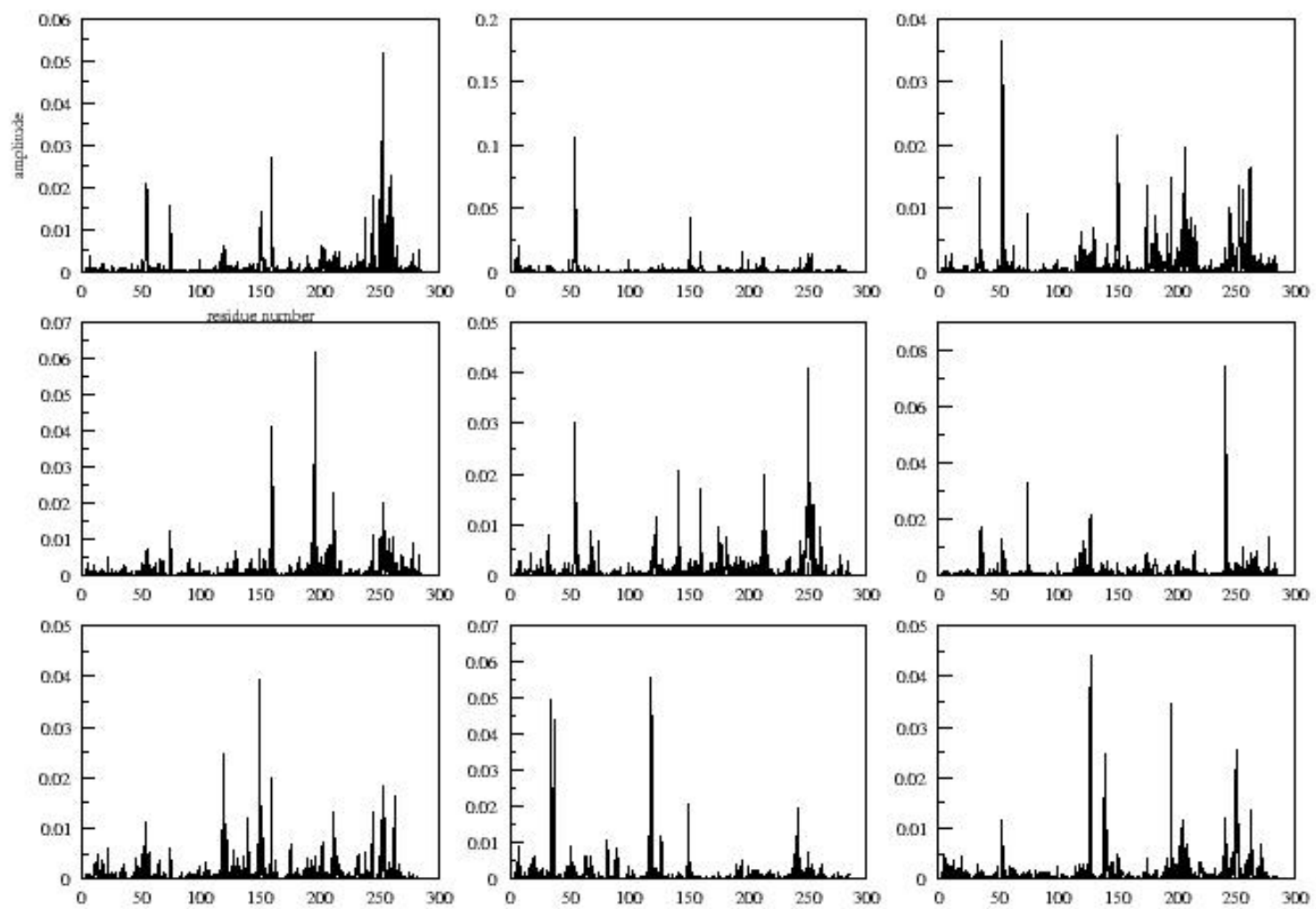

Figure 11a 

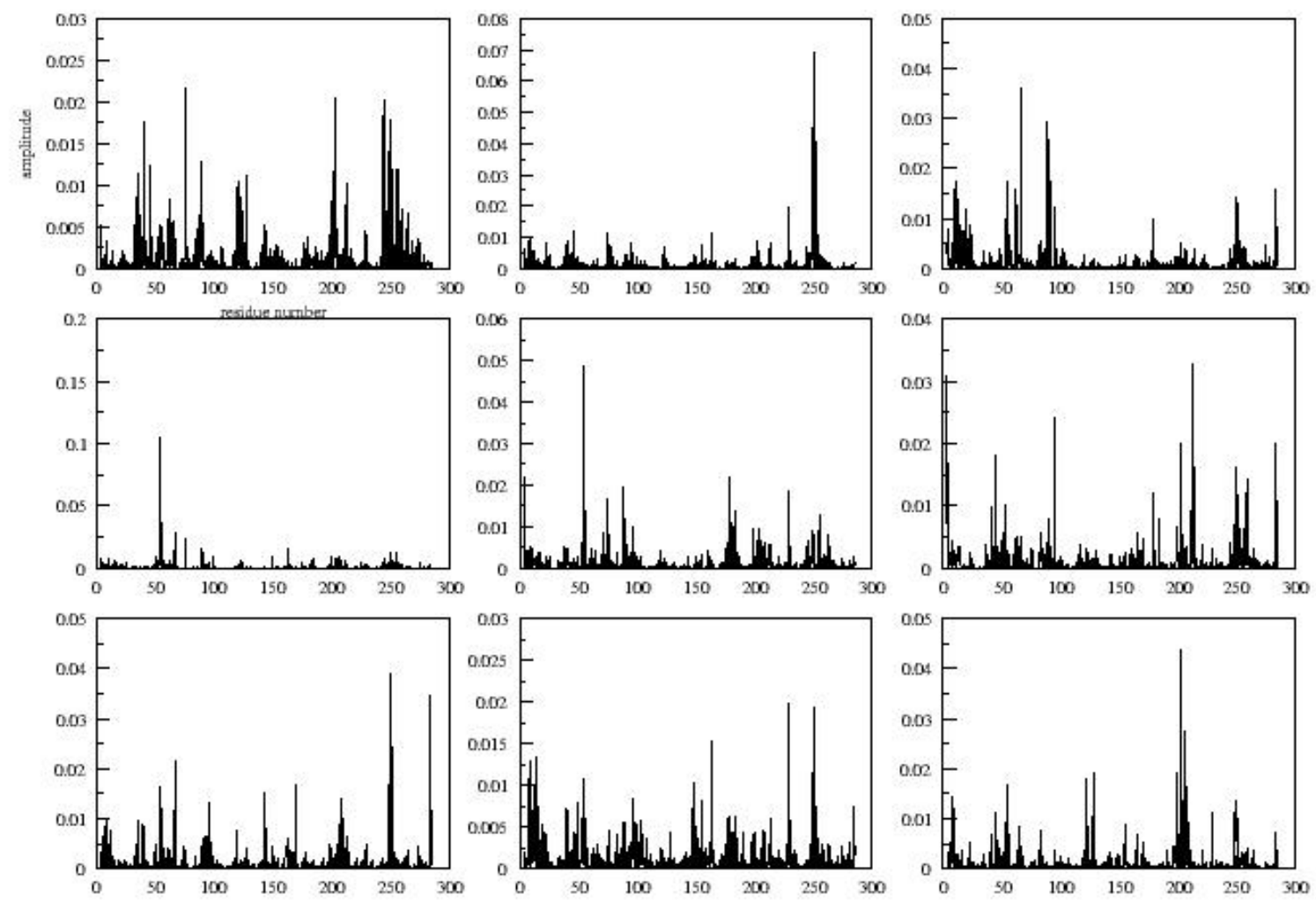

Figure 12a 

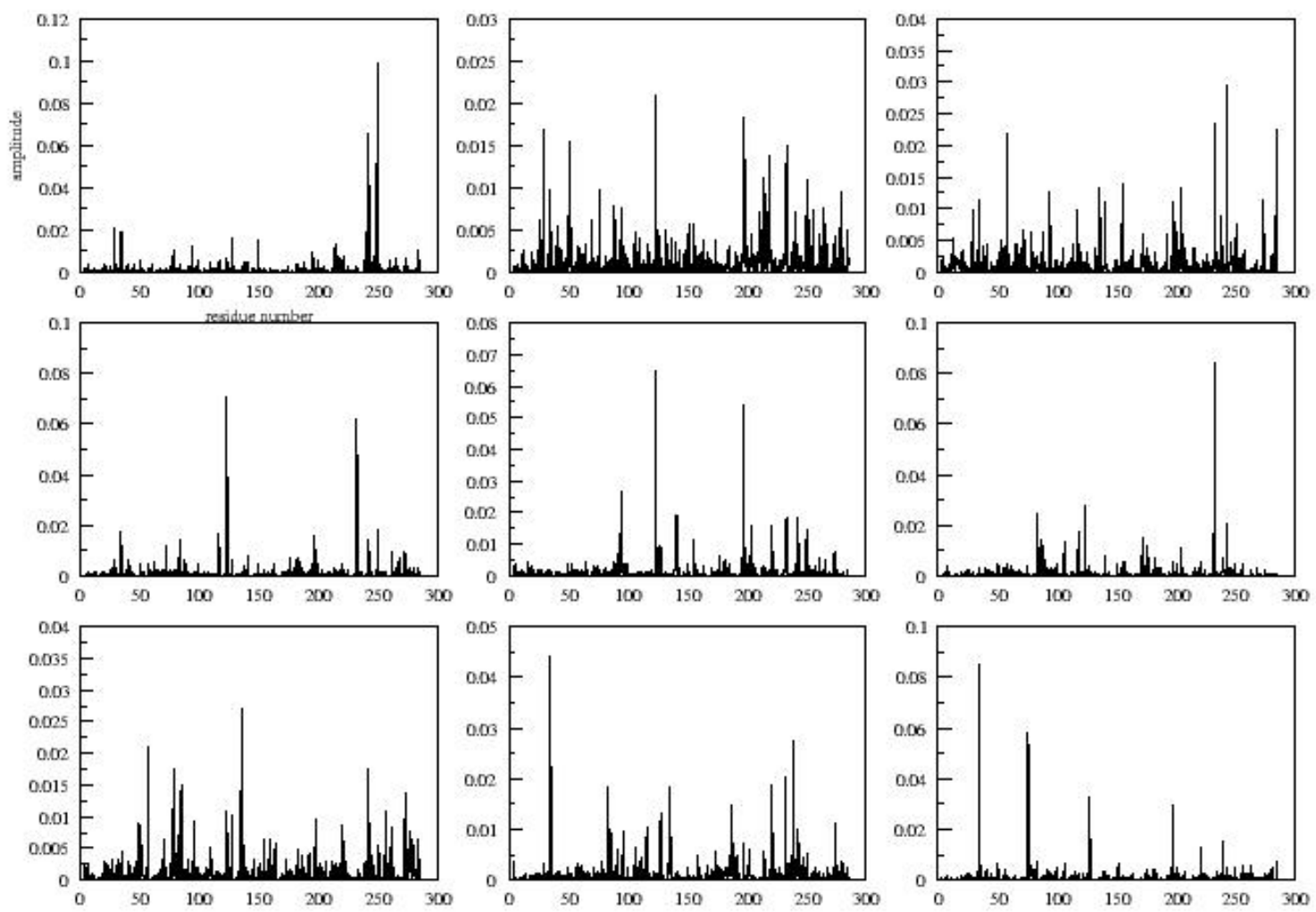

Figure 13a 